\title{
Potencial antioxidativo da lágrima de adultos jovens
}

\author{
Tear antioxidant potential in young adults
}

\author{
Patrícia Ioschpe Gus ${ }^{1}$ \\ Adriane Belló-Klein² \\ Susana Llesuy ${ }^{3}$ \\ Guilherme Goulart Quinto ${ }^{4}$ \\ Guilherme Herrmann Matos 5 \\ Samir Jacob Bechara ${ }^{6}$
}

\section{RESUMO}

Objetivo: Estudar os antioxidantes da lágrima humana, estimulada pelo corte de cebola e a possível influência dos hábitos de vida sobre estas medidas. Métodos: A amostra consistiu de dez adultos jovens, que responderam questionário sobre o hábito de fumar, de ingerir bebidas alcoólicas, frutas, vegetais e cereais, de usar vitaminas e/ou drogas e de praticar exercícios. O potencial reativo antioxidante total (TRAP) foi analisado por meio da quimioluminescência do luminol, a superóxido dismutase (SOD) foi medida pela inibição do piragolol e a absorbância de $\mathrm{H}_{2} \mathrm{O}_{2}$ a 240 nm foi utilizada para identificar a catalase. Resultados: A média $\pm \mathrm{DP}$ dos valores de potencial reativo antioxidante total foi $33,8 \pm 11,5 \mu \mathrm{M}$ e de superóxido dismutase foi $10,8 \pm 1,9 \mathrm{U} / \mathrm{mL}$. Não foi identificada atividade da catalase. Detectou-se associação entre a prática regular de exercícios e aumento nos valores de potencial reativo antioxidante total $(p=0,021)$, com diferença de $18,6 \mu \mathrm{M}$ entre os indivíduos que se exercitavam pelo menos uma vez por semana e os sedentários. Sexo masculino e aumento na concentração de potencial reativo antioxidante total também se associaram estatisticamente $(\mathrm{p}=0,013)$, com diferença de $16,3 \mu \mathrm{M}$ entre os sexos. Houve associação entre hábito tabágico e aumento na superóxido dismutase $(\mathrm{p}=0,041)$, com diferença de $3,3 \mathrm{U} / \mathrm{mL}$ entre fumantes de mais de cinco cigarros/dia e não fumantes. O uso de vitamina $\mathrm{C}$ também demonstrou associação com a superóxido dismutase $(\mathrm{p}=0,018)$; a diferença para os que tomavam vitamina $\mathrm{C}$ foi de 3,3 U/mL a mais. Conclusão: Os antioxidantes do lacrimejamento reflexo foram mensuráveis em adultos jovens, e diferentes variáveis parecem ter influenciado nos resultados.

Descritores: Radicais livres; Olho; Lágrima; Estresse oxidativo; Superóxido dismutase; Antioxidantes; Ácido ascórbico

\section{INTRODUÇÃO}

A toxicidade do oxigênio foi descrita quase que simultaneamente à descoberta deste elemento como componente químico do ar. A base de sua toxicidade foi estabelecida em 1871* por Paul Bert ${ }^{(1)}$. Avanços no mecanismo molecular das reações tóxicas só foram elucidados em meados do século passado, quando se percebeu a semelhança entre o efeito deletério da irradiação com raios X e a exposição ao oxigênio hiperbárico, propondo-se a participação dos intermediários da redução do oxigênio à água em ambos os $\operatorname{processos}^{(2)}$.

Radical livre se constitui num átomo ou grupo de átomos contendo um ou mais elétrons desemparelhados em sua órbita externa. Além dos radicais, outras combinações ou formas de apresentação compõem o grupo de espé-

*Paul Bert (1871) apud ${ }^{(1)}$ 
cies ativas de oxigênio, como o peróxido de hidrogênio e o oxigênio singulete ${ }^{(3)}$.

Para conservar a homeostasia do meio interno, a produção de espécies ativas de oxigênio é neutralizada pela de agentes antioxidantes endógenos não-enzimáticos e enzimáticos. Dentre os primeiros, destacamos os lipofílicos e os hidrofílicos, que exercem o seu efeito protetor ao reduzir a peroxidação lipídica das membranas celulares ${ }^{(4)}$. Embora a peroxidação lipídica possibilite a renovação da membrana, a biossíntese de prostaglandinas e leucotrienos, a fagocitose, a pinocitose e a lise de membranas intracelulares, o seu excesso pode causar alterações de permeabilidade, perda da função secretória e morte celular. Dentre as enzimas protetoras da oxidação, temos a superóxido dismutase (SOD), a catalase (CAT) e a glutationa peroxidase $(\mathrm{GPx})^{(1)}$.

O aumento na formação de espécies ativas de oxigênio ou a diminuição na defesa antioxidante é definido como estresse oxidativo, cujo envolvimento em muitas doenças é amplamente reconhecido. As células e os fluidos biológicos possuem um arsenal de mecanismos protetores contra as reações de oxidação, os quais agem prevenindo a produção de radicais livres ou reparando o dano oxidativo ${ }^{(5)}$.

Efeitos nocivos da poluição, do fumo, das radiações e de medicamentos podem levar à formação de radicais livres ${ }^{(1)}$. Inúmeros estudos experimentais já sugeriram a relação entre as espécies ativas de oxigênio e a fisiopatologia de muitas doenças, incluindo o enfisema pulmonar, a doença de Parkinson, a doença de Alzheimer, o câncer, a hipertensão arterial sistêmica, a aterosclerose, a degeneração macular relacionada à idade e a catarata ${ }^{(6)}$. Substâncias químicas exógenas têm sido testadas para neutralizar esses efeitos e também para minimizar as reações degenerativas do envelhecimento ${ }^{(7-8)}$.

A doença e as estruturas oculares mais estudadas com relação ao estresse oxidativo, são respectivamente, o glaucoma, a córnea, o cristalino, a retina e a úvea. As publicações consistem, em sua maioria, em protocolos com animais, porém, alguns estudos já foram realizados em humanos ${ }^{(7,9-10)}$.

A lágrima é estrutura vital para uma boa visão e, sendo ela de fácil acesso, este estudo preconiza estabelecer uma medida padrão de atividade antioxidante em adultos jovens, com risco muito baixo aos pacientes. Além disso, objetiva também verificar a influência dos hábitos de vida nos medidores de oxidação.

\section{MÉTODOS}

\section{Casuística}

Consistiu em estudantes do Curso de Medicina do sexto semestre da Universidade Luterana do Brasil que estivessem cursando a Disciplina de Sistema Visual.

\section{Critérios de seleção de amostra}

Foi oferecida a oportunidade de participar do estudo a todos os alunos da turma. Esta era composta somente por alunos caucasianos.
Os fatores de exclusão foram: idade inferior a 20 e superior a 30 anos, patologia sistêmica de caráter crônico-degenerativo, patologia ocular (incluiu-se somente os vícios refracionais), e os alunos que não conseguissem atingir o volume mínimo de 150 microlitros de lágrima após a segunda tentativa de corte da cebola.

\section{Método}

O lacrimejamento foi provocado pelo corte de cebola ${ }^{(9-10)}$. Foi aplicado um questionário com sete perguntas, visando o registro de hábitos alimentares, exercício físico, condições gerais de saúde, hábito de fumar, uso de medicamentos e de vitaminas.

Foi assinado também um termo de consentimento aprovado pela Comissão de Ética do Hospital das Clínicas de São Paulo.

A análise bioquímica foi realizada no Laboratório de Fisiologia Cardiovascular da Universidade Federal do Rio Grande do Sul, em Porto Alegre.

Inicialmente, pelo desconhecimento do volume necessário de lágrima para que as técnicas de rotina fossem sensíveis, estabeleceu-se a necessidade de projeto piloto. Nesse, realizouse coleta lacrimal de várias pessoas com pipeta esterilizada de fertilização "in vitro" de ponta romba, sem anestesia tópica. A lágrima de todos foi colocada num mesmo tubo descartável tipo "Eppendorf". A coleta foi feita do fundo-de-saco lacrimal e da que escorresse pelo rosto. $\mathrm{O}$ material coletado foi armazenado a - $20^{\circ} \mathrm{C}$ por uma semana. $\mathrm{O}$ descongelamento das amostras foi realizado em temperatura ambiente. O tempo de armazenamento e de descongelamento foi sempre o mesmo.

\section{Técnicas laboratoriais}

\section{Potencial antioxidante total (TRAP)}

Representa a capacidade antioxidante não enzimática do tecido, determinada através da medida da intensidade de quimioluminescência do luminol (LH2) induzida pela adição de 2, 2'-azo-bis (2-amidinopropano) (ABAP) à temperatura ambiente $^{(11)}$. O método se baseia no tempo de indução da oxidação de uma dispersão lipídica exposta ao ABAP, que representa fonte de radicais livres com taxa de produção constante e conhecida. O TRAP foi medido no contador beta (LKB Rack Beta Liquid Scintillation Specttometer-1215; LKB Produkter AB, Bromma, Swedem).

Para a contagem de quimioluminescência basal, adicionouse $4 \mu \mathrm{l}$ de solução de ABAP 10 mM em tampão glicina. A seguir, adicionou-se um volume de $10 \mu \mathrm{l}$ de solução de luminol $10 \mathrm{mM}$ à mistura anterior, também preparada em tampão glicina; esta representando a contagem inicial. A contagem inicial atinge um platô rapidamente, e assim permanece por vários minutos.

Acrescenta-se $10 \mu \mathrm{l}$ de Trolox 80 micromolar em tampão glicina (vitamina $\mathrm{E}$ hidrossolúvel, que funciona como padrãoouro) ou $10 \mu \mathrm{l}$ de lágrima (amostra teste). Ambas reduzem a intensidade de quimioluminescência do LH2 à medida que os antioxidantes vão sendo consumidos. O tempo necessário para que a contagem retorne ao valor inicial é proporcional à 
capacidade antioxidante da lágrima, e será comparado ao tempo de indução do Trolox. Os resultados são expressos em nmol de Trolox/mg de proteína.

\section{Superóxido dismutase}

A técnica utilizada para determinar a atividade da SOD se baseia na inibição da reação do radical superóxido pelo pirogalol $^{(1)}$, que é um composto que se auto-oxida com a variação de $\mathrm{pH}$. Em meio básico, a auto-oxidação do pirogalol gera superóxido. A SOD da lágrima compete com o sistema de detecção pelo radical superóxido.

Uma vez não sendo possível determinar a concentração da enzima nem sua atividade na forma de "substrato consumido/ tempo", utiliza-se uma unidade relativa. Define-se uma unidade de SOD como a quantidade de enzima necessária para inibir a velocidade de oxidação do detector em $50 \%$ do seu valor original.

A oxidação do pirogalol forma um produto colorido, detectado espectrofotometricamente a $420 \mathrm{~nm}$, durante dois minutos. Determina-se a atividade da SOD através da velocidade de formação do pirogalol oxidado ${ }^{(1)}$.

Foram utilizados $973 \mu \mathrm{l}$ de tampão tris $50 \mathrm{mM}(\mathrm{pH} 8,2), 8 \mu \mathrm{l}$ de pirogalol $24 \mathrm{mM}, 4 \mu \mathrm{l}$ de catalase e $30 \mu \mathrm{l}$ de lágrima. Utilizaram-se três distintas concentrações de $\operatorname{SOD}(0,25 \mathrm{U} ; 0,5 \mathrm{U}$ e $1 \mathrm{U})$ para a confecção da curva-padrão. A curva-padrão fornece a equação da reta para a realização dos cálculos, os quais foram expressos em U SOD/mL. As medidas de SOD foram realizadas no espectrofotômetro (Cary 1E, Varian, Autralia).

\section{Catalase}

A sua presença na amostra lacrimal foi pesquisada através da diminuição da absorbância de $\mathrm{H}_{2} \mathrm{O}_{2}$ a $240 \mathrm{~nm}^{(11)}$. As medidas da catalase foram também realizadas no espectrofotômetro (Cary 1E, Varian, Australia).

\section{Delineamento e análise estatística}

Trata-se de "survey" exploratório.

Os dados quantitativos foram descritos sob a forma de média e desvio padrão, enquanto as variáveis qualitativas foram descritas por freqüência e percentual. As variáveis de desfecho (TRAP e SOD) foram comparadas entre grupos formados pelas variáveis do questionário. As comparações foram feitas através da análise de variância, com localização de diferenças pelo teste de Duncan. Para explorar o efeito das variáveis do questionário sobre o desfecho, utilizou-se a técnica de regressão linear. Na situação onde foram comparadas variáveis dicotomizadas, utilizou-se o teste T de Student.

O nível de significância adotado foi de $\alpha=0,05$. Os dados foram processados e analisados com o auxílio do programa "Statistical Package for the Social Sciences" (SPSS), versão 10,0.

\section{RESULTADOS}

Dez sujeitos conseguiram completar o volume mínimo de lacrimejamento necessário para a análise. O volume mínimo individual estabelecido foi de $150 \mu \mathrm{l}$, conforme projeto piloto, o qual priorizou as medidas de TRAP, SOD e CAT. Cinco homens e 5 mulheres participaram do estudo final. Um dos homens não foi medido para SOD por problemas técnicos do laboratório.

A tabela 1 apresenta as características do questionário, onde se observa que a idade média encontra-se em torno de 23 anos. Observa-se igual número de homens e mulheres. Salienta-se a proporção de $50 \%$ de fumantes e $80 \%$ que alegam prática regular de exercícios. Quanto aos hábitos alimentares, $30 \%$ consomem dois ou mais porções de frutas por dia ou uma ou mais porções de vegetais por dia. O consumo de vitaminas somente é mencionado por $20 \%$, mas o de cereais por $50 \%$.

As médias $( \pm \mathrm{DP})$ encontradas para o TRAP e para a SOD foram, respectivamente, $33,8 \pm 11,5 \mu \mathrm{M}$ e 10,8 $\pm 1,9 \mathrm{U} / \mathrm{mL}$. Não foi possível detectar atividade da catalase.

As diferenças mais importantes observadas nas concentrações do TRAP estavam associadas ao sexo $(\mathrm{p}=0,013)$ e à prática de exercícios ( $\mathrm{p}=0,055)$ (Tabela 2$)$. As outras variáveis estudadas não apresentaram associações relevantes com o TRAP.

\begin{tabular}{|c|c|}
\hline \multicolumn{2}{|l|}{ Característica } \\
\hline Idade (anos) & $23,1 \pm 1,7$ \\
\hline \multicolumn{2}{|l|}{ Sexo } \\
\hline Masculino & 5 \\
\hline Feminino & 5 \\
\hline \multicolumn{2}{|c|}{ Fumo (cigarro/dia) } \\
\hline Nunca & 5 \\
\hline$\leq 5$ & 2 \\
\hline$>5$ & 3 \\
\hline \multicolumn{2}{|c|}{ Álcool (doses/dia) } \\
\hline Nunca & 2 \\
\hline$<1$ & 8 \\
\hline$\geq 1$ & 0 \\
\hline \multicolumn{2}{|l|}{ Frutas ( $\mathrm{n} \%$ dia) } \\
\hline Nunca & 1 \\
\hline$<2$ & 6 \\
\hline$\geq 2$ & 3 \\
\hline \multicolumn{2}{|c|}{ Verdes (pratos/dia) } \\
\hline Nunca & 1 \\
\hline$<1$ & 6 \\
\hline$\geq 1$ & 3 \\
\hline \multicolumn{2}{|c|}{ Exercícios (vezes/semana) } \\
\hline Nunca & 2 \\
\hline$<3$ & 5 \\
\hline 3 & 3 \\
\hline$\geq 3$ & 0 \\
\hline \multicolumn{2}{|l|}{ Vitaminas } \\
\hline Não & 8 \\
\hline Sim & 2 \\
\hline \multicolumn{2}{|l|}{ Cereais } \\
\hline Não & 5 \\
\hline Sim & 5 \\
\hline $\begin{array}{l}\text { Os dados são a } \\
\text { absoluta. }\end{array}$ & e freqüência \\
\hline
\end{tabular}


O fator de maior destaque em associação com a SOD foi o hábito tabágico. Este último apresentou significância limítrofe $(\mathrm{p}=0,076)$, enquanto a análise de regressão linear detectou uma tendência para o aumento da SOD no maior consumo de cigarros $(\mathrm{p}=0,041)$. Quanto aos outros fatores, não foram observadas associações importantes com a SOD (Tabela 3).

\section{DISCUSSÃO}

Em função das reações fotooxidativas e de toxinas químicas, a superfície e o compartimento anterior do olho estão constantemente expostos à ação das espécies ativas de oxigênio. A superfície ocular também está envolvida na proteção antiinfecciosa, sendo os leucócitos ativados uma importante fonte de radicais ${ }^{(12)}$. Os antioxidantes auxiliam na resposta inflamatória, na cicatrização corneana e mantêm a estabilidade do filme lacrimal ${ }^{(10)}$.

O presente estudo optou por estudar o potencial reativo antioxidante total (TRAP) e as enzimas. As últimas, por ordem de prioridade na cascata antioxidante. Começou-se pela SOD,

\begin{tabular}{|c|c|c|c|}
\hline Característica & $\mathbf{N}$ & TRAP $(\mu M)$ & $\mathbf{P}$ \\
\hline \multicolumn{4}{|l|}{ Sexo } \\
\hline Masculino & 5 & $41,9 \pm 7,6$ & \multirow[t]{2}{*}{0,013} \\
\hline Feminino & 5 & $25,6 \pm 8,6$ & \\
\hline \multicolumn{4}{|c|}{ Fumo (cigarros/dia) } \\
\hline Nunca & 5 & $34,6 \pm 13,7$ & \multirow{3}{*}{0,978} \\
\hline$\leq 5$ & 2 & $32,6 \pm 9,2$ & \\
\hline$>5$ & 3 & $33,2 \pm 3,2$ & \\
\hline \multicolumn{4}{|c|}{ Álcool (doses/dia) } \\
\hline Nunca & 2 & $44,1 \pm 7,1$ & \multirow{3}{*}{0,170} \\
\hline$<1$ & 8 & $31,2 \pm 11,2$ & \\
\hline$\geq 1$ & 0 & 0 & \\
\hline \multicolumn{4}{|l|}{ Frutas ( $\mathrm{n} \% / \mathrm{dia})$} \\
\hline Nunca & 2 & $29,8 \pm 5,3$ & \multirow{3}{*}{0,148} \\
\hline$<2$ & 6 & $39,1 \pm 11,1$ & \\
\hline$\geq 2$ & 2 & $21,6 \pm 6,9$ & \\
\hline \multicolumn{4}{|c|}{ Verdes (pratos/dia) } \\
\hline Nunca & 1 & $33,7 \pm 0,0$ & \multirow{3}{*}{0,559} \\
\hline$<1$ & 6 & $37,0 \pm 12,3$ & \\
\hline$\geq 1$ & 3 & $27,4 \pm 11,2$ & \\
\hline \multicolumn{4}{|c|}{ Exercícios (vezes/semana) } \\
\hline Nunca & 2 & $18,3 \pm 2,2$ & \multirow{4}{*}{0,055} \\
\hline$<3$ & 5 & $39,9 \pm 6,5$ & \\
\hline 3 & 3 & $33,9 \pm 13,2$ & \\
\hline$\geq 3$ & 0 & 0 & \\
\hline \multicolumn{4}{|l|}{ Vitaminas } \\
\hline Não & 8 & $34,0 \pm 11,0$ & \multirow[t]{2}{*}{0,922} \\
\hline Sim & 2 & $33,0 \pm 18,7$ & \\
\hline \multicolumn{4}{|l|}{ Cereais } \\
\hline Não & 5 & $35,0 \pm 10,6$ & \multirow[t]{2}{*}{0,750} \\
\hline Sim & 5 & $32,5 \pm 13,5$ & \\
\hline
\end{tabular}

a qual bloqueia o radical superóxido, o primeiro a ser formado, seguindo-se pela CAT, que reage com o peróxido de hidrogênio, e por último, a GPx. Em função da dificuldade na obtenção do volume de lágrima, só se conseguiu testar até a CAT.

Questionou-se se a falta de detecção da CAT ocorreu pela sua real inexistência ou pelo pequeno volume de lágrima que sobrou para a sua reação, visto que antes foram testados o TRAP e a SOD. Alguns autores estudaram amostras basais de lágrima de 3 a $8 \mu$ de 13 indivíduos saudáveis entre 20 e 64 anos à procura de SOD, CAT e GPx. Eles detectaram a presença de SOD, mas também não encontraram atividade da CAT nem da $\mathrm{GPX}^{(13)}$.

Pela falta de literatura oftalmológica que valorize os aspectos abaixo explorados, utilizou-se dados da bibliografia médica de outras especialidades como fonte da discussão.

\section{Sexo e TRAP}

O presente estudo detectou que os homens apresentaram concentrações de TRAP lacrimal superior às das mulheres

\begin{tabular}{|c|c|c|c|}
\hline Característica & $\mathbf{N}^{*}$ & SOD (U/ml) & $\mathbf{P}$ \\
\hline \multicolumn{4}{|l|}{ Sexo } \\
\hline Masculino & 4 & $10,8 \pm 3,0$ & 0,940 \\
\hline Feminino & 5 & $10,7 \pm 0,7$ & \\
\hline \multicolumn{4}{|c|}{ Fumo (cigarros/dia) } \\
\hline Nunca & 5 & $10,0 \pm 1,3$ & \\
\hline$\leq 5$ & 2 & $10,2 \pm 0,4$ & 0,076 \\
\hline$>5$ & 2 & $13,3 \pm 2,4$ & \\
\hline \multicolumn{4}{|c|}{ Álcool (doses/dia) } \\
\hline Nunca & 2 & $10,2 \pm 0,4$ & \\
\hline$<1$ & 7 & $10,2 \pm 2,2$ & 0,643 \\
\hline$\geq 1$ & 0 & 0 & \\
\hline \multicolumn{4}{|l|}{ Frutas (n/dia) } \\
\hline Nunca & 1 & $10,5 \pm 0,0$ & \\
\hline$<2$ & 6 & $10,8 \pm 2,4$ & 0,992 \\
\hline$\geq 2$ & 2 & $10,8 \pm 0,5$ & \\
\hline \multicolumn{4}{|c|}{ Verdes (pratos/dia) } \\
\hline Nunca & 1 & $10,1 \pm 0,0$ & \\
\hline$<1$ & 5 & $11,1 \pm 2,6$ & 0,887 \\
\hline$\geq 1$ & 3 & $10,5 \pm 0,6$ & \\
\hline \multicolumn{4}{|c|}{ Exercícios (vezes/semana) } \\
\hline Nunca & 2 & $11,4 \pm 0,4$ & \\
\hline$<3$ & 4 & $10,7 \pm 3,0$ & 0,897 \\
\hline 3 & 3 & $10,4 \pm 0,1$ & \\
\hline$\geq 3$ & 0 & 0 & \\
\hline \multicolumn{4}{|l|}{ Vitaminas } \\
\hline Não & 7 & $10,0 \pm 1,1$ & 0,018 \\
\hline Sim & 2 & $13,3 \pm 2,4$ & \\
\hline \multicolumn{4}{|l|}{ Cereais } \\
\hline Não & 4 & $10,5 \pm 0,8$ & 0,744 \\
\hline Sim & 5 & $11,0 \pm 2,6$ & \\
\hline
\end{tabular}


$(\mathrm{p}=0,013)$. As diferenças no estresse oxidativo entre homens e mulheres costumam ser atribuída à ação hormonal. Quando interagem outras variáveis como o hábito de fumar e o condicionamento físico, as diferenças se acentuam e até se contradizem.

Outros autores estudaram a diferença de suscetibilidade ao estresse oxidativo causado pelo hábito de fumar entre homens e mulheres ${ }^{(14)}$. Eles observaram que, durante o mesmo período de exposição, mulheres com até 50 anos desenvolveram níveis mais altos de anticorpos anti-5-hidroximetil-2'-deoxiuridina e atingiram picos mais altos com menos anos de exposição (30 anos) do que os homens (40 anos) da mesma idade. $\mathrm{O}$ achado sugere interações hormonais e seus efeitos no aumentado risco de câncer de pulmão em fumantes comparadas aos fumantes masculinos.

O processo de envelhecimento está relacionado à ação dos radicais livres. Para investigar a possível associação entre os níveis de antioxidantes plasmáticos e o desempenho cognitivo em idosos saudáveis, Perrig et al., estudaram 442 indivíduos de 65 a $94 \operatorname{anos}^{(15)}$. Eles foram testados para capacidade de memória, níveis de $\alpha$-tocoferol, acido ascórbico e betacaroteno, colesterol, ferritina, e medida da pressão arterial sistêmica em 1971 e em 1993. Houve significativa estabilidade dos antioxidantes plasmáticos durante os 22 anos, sendo que a memória, o reconhecimento e o vocabulário se correlacionaram significativamente com os níveis de ácido ascórbico e beta-caroteno. Não houve diferença entre os sexos na performance cerebral dos idosos neste estudo.

\section{Exercícios e TRAP}

Observou-se, neste estudo, que a prática regular de exercício por pelo menos uma vez por semana se relacionou ao aumento das concentrações de TRAP lacrimal em comparação com sedentários $(\mathrm{p}=0,021)$. Questiona-se se, em se tratando de amostra com faixa etária homogênea e jovem, esta diferença não sofreria a influência do hábito de exercícios ter sido subjetivamente mais regular entre os homens.

A literatura não é sempre concordante em relação aos benefícios da prática esportiva em diminuir o estresse oxidativo. Se realizado infreqüentemente, o exercício não modifica o perfil antioxidante, ou até gera aumento agudo na lipoperoxidação ${ }^{(16)}$. Adquirindo regularidade, entretanto, tende a baixar as gorduras do sangue e a aumentar a resistência antioxidante ${ }^{(17-19)}$.

A relação entre atividade física e TRAP foi avaliada no "Nosthern Ireland Health and Activity Survey", um estudo populacional transversal que estudou 1.600 indivíduos. A média de TRAP sérico foi significativamente mais alta nos homens do que nas mulheres. Tanto os fumantes masculinos quanto os femininos tiveram TRAP mais baixo do que os não fumantes ${ }^{(20)}$. Os autores acreditam que os potenciais benefícios do exercício não se relacionam com aumento no potencial antioxidante, tanto nos homens quanto nas mulheres.

\section{Hábito tabágico e SOD}

O fumo causa aumento nas espécies ativas de oxigênio e de nitrogênio. Os estudos têm demonstrado que a combustão de oxidantes presentes no cigarro aumenta o estresse oxidativo das vias aéreas, da respiração, do sangue e da urina de fumantes testados. Isso provoca inativação de antiproteinases, lesão epitelial das vias aéreas, aumento de neutrófilos na microvasculatura pulmonar e expressão genética de mediadores pró-inflamatórios ${ }^{(21)}$.

O presente estudo revelou maiores concentrações de SOD lacrimal no grupo que consumia mais de cinco cigarros por dia, quando comparado com o grupo que consumia menos de cinco cigarros por dia. Poder-se-ia especular que a superfície ocular estando completamente exposta à fumaça de cigarro, as pessoas tenderiam a adquirir maior resistência antioxidante à maior agressão.

\section{Vitaminas e SOD}

Apenas 2 dos 9 alunos testados para a SOD faziam uso regular de vitaminas, no caso, o ácido ascórbico. Houve perceptível tendência ao aumento das concentrações de SOD nestes dois casos. Todos os outros não utilizavam reposição vitamínica.

A vitamina $\mathrm{C}$ é o principal interruptor do processo de lipoperoxidação da superfície ocular, além de inibir a atividade dos polimorfonucleares por danificar a mieloperoxidase dos tecidos inflamados ${ }^{(22)}$. É também fundamental para a síntese de colágeno ${ }^{(23)}$.

A literatura ocular sugere que uma dieta rica em proteína, vitaminas A, B6 e C, potássio e zinco, é necessária para a função lacrimal normal ${ }^{(24)}$. Excessos de gordura, sal, colesterol, álcool, proteínas e açúcares têm sido associados a causas de disfunção lacrimal ${ }^{(25)}$.

\section{CONCLUSÕES}

Existe mensurável atividade do TRAP e da SOD na lágrima estimulada de adultos jovens e saudáveis. O sexo masculino e a prática regular de exercícios por pelo menos uma vez por semana se relacionaram ao aumento nos valores de TRAP, enquanto o hábito tabágico e a ingestão de vitamina $\mathrm{C}$ aumentaram a atividade de SOD lacrimal.

O presente estudo se propôs a fornecer valores lacrimais de sujeitos jovens e normais, úteis para o futuro estudo de condições patológicas. É importante observar, entretanto, que as características ambientais, demográficas e comportamentais de futuras amostras populacionais devem ser semelhantes para que os resultados aqui coletados sejam proveitosos. Estudos epidemiológicos devem ser realizados para confirmar as associações encontradas neste estudo.

\section{ABSTRACT}

Purpose: To study the antioxidant status of human tears, stimulated by onion fumes and the possible influence of the life habits thereon were measured. Methods: Subjects were ten healthy young adults, who answered a questionnaire 
about smoking, alcohol ingestion, fruit, vegetable, cereal, and vitamin intake and/or intake of other drugs, and physical exercise habits. Chemoluminescensce of luminol was used to analyze the total reactive antioxidant potential (TRAP), inhibition of piragollol was used to measure superoxide dismutase (SOD) and absorbance of $\mathrm{H}_{2} \mathrm{O}_{2}$ at $240 \mathrm{~nm}$ was used to identify catalase. Results: Mean $\pm \mathrm{SD}$ value for total reactive antioxidant potential was $33.8 \pm 11.5 \mu \mathrm{M}$ and for superoxide dismutase $10.8 \pm 1.9 \mathrm{U} / \mathrm{mL}$. Catalase was not identified. Regular exercise was associated with increased total reactive antioxidant potential values $(\mathrm{p}=0.021)$, with a difference of $18.6 \mu \mathrm{M}$ between individuals who exercise at least once a week and sedentary individuals. Male gender and total reactive antioxidant potential values were statistically associated $(\mathrm{p}=0.013)$, with a difference of $16.3 \mu \mathrm{M}$ between genders. There was an association between smoking and increased superoxide dismutase values $(\mathrm{p}=0.041)$, with a difference of $3.3 \mathrm{U} / \mathrm{mL}$ between smokers of more than five cigarettes/day and non-smokers. Also, vitamin C intake and superoxide dismutase values were associated $(\mathrm{p}=0.018)$; the difference for vitamin $\mathrm{C}$ takers was $3.3 \mathrm{U} / \mathrm{mL}$. Conclusion: Reflex tearing antioxidants were measurable in healthy young adults, and different variables apparently influenced their values.

Keywords: Free radicals; Eye; Tears; Oxidative stress; Superoxide dismutase; Antioxidants; Ascorbic acid

\section{REFERÊNCIAS}

1. Belló-Klein A. Ação da vitamina E e do trolox sobre o estresse oxidativo produzido pelo peróxido de hidrogênio no miocárdio isolado de rato [tese]. Porto Alegre: Faculdade de Medicina, Universidade Federal do Rio Grande do Sul; 1993.

2. Gerschman R, Gilbert DL, Nye SW, Dwyer P, Fenn WO. Oxygen poisoning and X-irradiation: a mechanism in common. Science. 1954;119(3097):623-6.

3. Southorn PA, Powis G. Free radicals in medicine I. Chemical nature and biologic reactions. Mayo Clin Proc. 1988;63(4):381-9.

4. Sies H, Murphy ME. Role of tocopherols in the protection of biological systems against oxidative damage. J Photochem Photobiol Biol. 1991;8(2):211-8.

5. Koracevic D, Koracevic G, Djordjevic V, Andrejevic S, Cosic V. Method for the measurement of antioxidant activity in human fluids. J Clin Pathol. 2001; 54(5):356-61. Comment in: J Clin Pathol. 2001;54(5):339.
6. Halliwell B, Gutteridge JC. Free radicals in biology and medicine. 3rd ed. Oxford, London: Clarendon; 1999.

7. Green K. Free radicals and aging of anterior segment tissues of the eye: a hypothesis. Ophtalmic Res. 1995;27(Suppl 1):143-9.

8. Ward JA. Should antioxidant vitamins be routinely recommended for older people? Drugs Aging. 1998;12(3):169-75.

9. Kuizenga A, Van Haeringen NJ, Kijlstra A. Inhibition of hydroxyl radical formation by human tears. Invest Ophthalmol Vis Sci. 1987;28(2):305-13.

10. Choy CK, Benzie IF, Cho P. Ascorbic acid concentration and total antioxidant activity of human tear fluid measured using the FRASC assay. Invest Ophthalmol Vis Sci. 2000;41(11):3293-8.

11. Boveris A, Chance B. The mitochondrial generation of hydrogen peroxide. General properties and effect of hyperbaric oxygen. Biochem J. 1973;134(3): 707-16.

12. Augustin AJ, Spitznas M, Kaviani N, Meller D, Koch FH, Grus F, et al. Oxidative reactions in the tear fluid of patients suffering from dry eyes. Graefe's Arch Clin Exp Ophthalmol. 1995;233(11):694-8.

13. Crouch RK, Goletz P, Snyder A, Coles WH. Antioxidant enzymes in human tears. J Ocul Pharmacol. 1991;7(3):253-8.

14. Mooney LA, Perera FP, Van Bennekum AM, Blaner WS, Karkoszka J, Covey $\mathrm{L}$, et al. Gender differences in autoantibodies to oxidative DNA base damage in cigarette smokers. Cancer Epidemiol Biomarkers Prev. 2001;10(6):641-8.

15. Perrig WJ, Perrig P, Stahelin HB. The relation between antioxidants and memory performance in the old and very old. J Am Geriatr Soc. 1997;45(6): 718-24.

16. Tidus PM. Estrogen and gender effects on muscle damage, inflammation, and oxidative stress. Can J Appl Physiol. 2000;25(4):274-87.

17. Vasankari TJ, Kujala UM, Vasankari TM, Ahotupa M. Reduced oxidized LDL levels after a 10-month exercise program. Med Sci Sports Exerc. 1998;30(10): 1496-501.

18. Kotska T, Drai J, Berthouze SE, Lacour JR, Bonnefoy M. Physical activity, aerobic capacity and selected markers of oxidative stress and the anti-oxidant defense system in healthy active elderly men. Clin Physiol. 2000;20(3):185-90.

19. Ginsburg GS, O’Toole M, Rimm E, Douglas PS, Rifai N. Gender differences in exercise-induced changes in sex hormone levels and lipid peroxidation in athletes participating in the Hawaii Ironman triathlon. Ginsburg-gender and exercise-induced lipid peroxidation. Clin Chim Acta. 2001;305(1-2):131-9.

20. Sharpe PC, Duly EB, MacAuley D, McCrum EE, Mulholland C, Stott G, et al. Total radical trapping antioxidant potential (TRAP) and exercise. QJM. 1996;89(3):223-8.

21. McNee W. Oxidants/antioxidants and COPD. Chest. 2000;117(5 Suppl 1): 303S-17S.

22. Kasetsuwan N, Wu FM, Hsieh F, Sanchez D, McDonnell P. Effect of topical ascorbic acid on free radical tissue damage and inflammatory cell influx in the cornea after excimer laser corneal surgery. Arch Ophthalmol. 1999;117(5):649-52.

23. Bilgihan A, Bilgihan K, Toklu Y, Konuk O, Yis O, Hasanreisoglu B. Ascorbic acid levels in human tears after photorefractive keratectomy, transepithelial photorefractive keratectomy, and laser in situ keratomileusis. J Cataract Refract Surg. 2001;27(4):585-8.

24. Patel S, Plaskow J, Ferrier C. The influence of vitamins and trace element supplements on the stability of the pre-corneal tear film. Acta Ophthalmol (Copenh). 1993;71(6):825-9.

25. Caffery BE. Influence of diet on tear function. Optom Vis Sci. 1991;68(1):58-72.

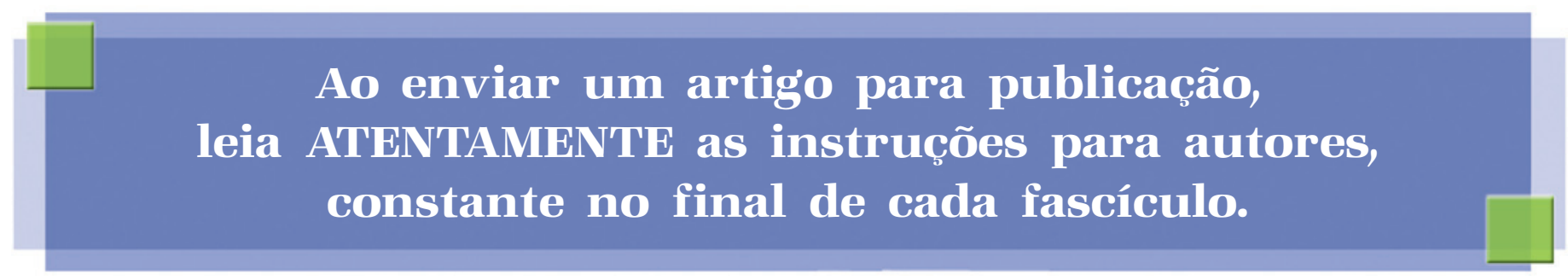

